\title{
Formidable Challenges in the Search for Biomarkers of Psychiatric Disorders
}

\section{Chad A. Bousman* and lan P. Everall}

Psychiatry, University of Melbourne, Parkville, Victoria, Australia

Discovery of a biomarker(s) for major mental illnesses such as schizophrenia, bipolar, and major depression would revolutionize psychiatry. The relatively recent beginning of the "omics" era has inspired psychiatric researchers to investigate molecular profiles of patients with psychiatric disorders in an effort to uncover distinct genotypic variation, expression, translation, and/or perturbed molecular networks that could then be used for screening, diagnosis, staging, and/or prognosis of psychiatric illness [1]. However, to date, the road to discovery has been quite turbulent and several major challenges have hindered discovery efforts.

The Achilles' heal of psychiatric biomarker research is the satisfactory phenotypic classification of research participants. Classification of major psychiatric disorders can have considerable flexibility, which can result in heterogeneity within a sample, potentially concealing underlying biological mechanisms and making comparisons between biomarker studies difficult. Classification of major psychiatric disorders depends on a set of subjective criteria used to assign individuals to a diagnostic category based on relevant clinical similarities. Major psychiatric disorder classification systems are polythetic in that two individuals may have the same phenotypic classification but exhibit or report variations in characteristic symptoms, in contrast to monothetic, in which the members would be identical in all characteristics [2]. Further complicating the classification of psychiatric disorders is the fact that there are two widely used classification systems: 1) Diagnostic and Statistical Manual of Mental Disorders (DSM-IV) [3] and 2) International Classification of Diseases (ICD-10) [4]. While these classification systems stem from similar scientific and conceptual roots, clinical nuances of major psychiatric disorders are discordant in the two systems despite attempts to unify them. This classification challenge ultimately leads to an inherent heterogeneity within and across studies that results in difficulties in replication of candidate biomarkers. To address this challenge is remarkably difficult as current classification systems are deeply embedded in clinical training and practice. However, there is hope that new versions of both the DSM and ICD classification systems, which are currently under development, will make refinements to address these limitations in phenotypic classification and in so doing improve psychiatric biomarker discovery efforts.

Another major challenge in psychiatric biomarker discovery is accounting for the effect of psychotropic medications. Previous research has shown that psychotropic medications (e.g. antipsychotics, moodstabilizers) can alter mRNA [5], miRNA [6] as well as metabolomic [7] and presumably proteomic profiles. Current research statistically adjusts for medication exposure when conducting analysis, this is typically done quite crudely (e.g. lifetime dose reported by patient) and thus the extent of the effects that medication has on molecular profiles is largely unknown. Our research group and others have utilized in vitro studies in order to tackle this problem by exposing a variety of human cultured cells to psychotropic medications preceded and followed by 'omic' analysis $[8,9]$. However, cell models, although informative, lack the complexity of normal in vivo central nervous system organ environment and as a result are not necessarily representative of what occurs in the human body. The ideal solution is recruitment of medication-naïve or first-episode patients into biomarker discovery research. The challenge is that these patients are difficult to identify and recruit in a timely and ethical manner.

Discovery of biomarkers for major psychiatric disorders will not only require addressing the above mentioned challenges but will also in part require a paradigm shift in biomarker discovery approach. Currently, the field has adopted a traditional reductionist approach in which focus is given to the examination of individual parts and their links to a complex psychiatric condition. Although this approach has enriched our understanding of major psychiatric disorders and assisted in narrowing our search for viable biomarkers, it is overly simplistic in its ability to provide robust biomarkers for complex phenomena in a variety of contexts. A progressive shift to a systems-thinking approach is required. Systems-thinking involves examination of all the individual constituents of complex phenomena such as major psychiatric disorders in the context of relationships with each other and other systems, rather than in isolation [10]. Indeed, psychiatric disorders cannot be explained by single factors and thus others have called for a broader, systems-biology view of psychiatric disorders integrating multiple "omics" and environmental factors and interactions [11]. A systems-biology approach emphasizes complex integration of the transcriptome, metabolome, proteome, and interactome, accounting for the context in which these networks operate [12]. Of course, our incomplete understanding of the individual constituents of the genome and other "omes", as well as technological limitations in examining data from disparate sources relevant to major psychiatric disorders and costs of existing technology obstruct the emergence of this paradigm shift. Nevertheless, tools concordant with the systemsthinking approach such as DOME (database for omes) [13], capable of storing functional genomics, proteomic, and metabolomic data in a single database, are being developed and will likely be transferable to biomarker-discovery efforts in major psychiatric disorders. The further development, availability, and use of these databases in the future will hasten integration of data from varying "omics" domains and improve our ability to identify biomarkers for psychiatric disorders.

Achieving this vision requires that new psychiatric biomarker discovery efforts continue pushing forward with innovative and sound methodological approaches, addressing challenges discussed here. The "perfect" biomarker discovery study in psychiatric disorders

*Corresponding author: Chad A. Bousman, University of Melbourne, Department of Psychiatry, Melbourne Brain Centre, Level 4, Victoria, 3010, Australia, E-mail: cbousman@unimelb.edu.au

Received August 21, 2011; Accepted October 23, 2011; Published October 25, 2011

Citation: Bousman CA, Everall IP (2011) Formidable Challenges in the Search for Biomarkers of Psychiatric Disorders. Metabolomics 1:105e. doi:10.4172/21530769.1000105e

Copyright: (C) 2011 Bousman CA, et al. This is an open-access article distributed under the terms of the Creative Commons Attribution License, which permits unrestricted use, distribution, and reproduction in any medium, provided the original author and source are credited. 
Citation: Bousman CA, Everall IP (2011) Formidable Challenges in the Search for Biomarkers of Psychiatric Disorders. Metabolomics 1:105e. doi:10.4172/2153-0769.1000105e

Page 2 of 2

may be unattainable. However, improved classification methods, innovative study design, technological advancements, and accelerated collaborative efforts between investigators will enhance our ability to discover and apply biomarkers to major psychiatric disorders.

\section{References}

1. Biomarkers Definitions Working Group (2001) Biomarkers and surrogate endpoints: preferred definitions and conceptual framework. Clin pharmaco Ther 69: 89-95

2. Millon T (1991) Classification in psychopathology: rationale, alternatives, and standards. J Abnorm Psychol 100: 245-261.

3. American Psychiatric A (1994) Diagnostic criteria from DSM-IV. Washington DC The Association 358.

4. World Health O (1992) ICD-10: international statistical classification of diseases and related health problems, 10th revision. Geneva: World Health Organization.

5. Chana G, Lucero G, Salaria S, Lozach J, Du P, et al. (2009) Upregulation of NRG-1 and VAMP-1 in human brain aggregates exposed to clozapine. Schizophr Res 113: 273-276.

6. Chen H, Wang N, Burmeister M, Mclnnis MG (2009) MicroRNA expression changes in lymphoblastoid cell lines in response to lithium treatment. Int $\mathrm{J}$ Neuropsychopharmacol 12: 975-981.
7. Kaddurah-Daouk R, McEvoy J, Baillie RA, Lee D, Yao JK, et al. (2007) Metabolomic mapping of atypical antipsychotic effects in schizophrenia. Mo psychiatry 12: 934-945.

8. Lee JG, Cho HY, Park SW, Seo MK, Kim YH (2010) Effects of olanzapine on brain-derived neurotrophic factor gene promoter activity in SH-SY5Y neuroblastoma cells. Prog Neuropsychopharmacol Biol Psychiatry 34: 10011006.

9. Chana G, Lucero G, Salaria S, Lozach J, Du P, et al. (2009) Upregulation of NRG-1 and VAMP-1 in human brain aggregates exposed to clozapine. Schizophr Res 113: 273-276.

10. Capra F, Lamantia P (1997) The web of life : a new scientific understanding of living systems. Vol. 1 Anchor Books trade paperback. New York, Anchor Books 347.

11. Reckow S, Gormanns P, Holsboer F, Turck CW (2008) Psychiatric disorders biomarker identification: from proteomics to systems biology. Pharmacopsychiatry 41 Suppl 1: S70-77.

12. Boogerd FC (2007) Systems biology : philosophical foundations. Amsterdam Boston: Elsevier 342

13. Mehrotra B, Mendes P (2006) Bioinformatics Approaches to Integrate Metabolomics and Other Systems Biology Data. Plant Metabolomics 57: 105115. 